\title{
Physical Layer Algorithm and Hardware Verification of MIMO Relays Using Cooperative Partial Detection
}

\author{
Kiarash Amiri, Michael Wu, Melissa Duarte, Joseph R. Cavallaro \\ Department of Electrical and Computer Engineering \\ MS-366, Rice University, 6100 Main St., Houston, TX 77005 \\ \{kiaa, mbw2, mduarte, cavallar\}@ rice.edu
}

\begin{abstract}
Cooperative communication with multi-antenna relays can significantly increase the reliability and speed. However, cooperative MIMO detection would impose considerable complexity overhead onto the relay if a full detect-and-forward (FDF) strategy is employed. In order to address this challenge, we propose a novel cooperative partial detection (CPD) strategy to partition the detection task between the relay and the destination. CPD utilizes the inherent structure of the tree-based sphere detectors, and modifies the tree traversal so that instead of visiting all the levels of the tree, only a subset of the levels, thus a subset of the transmitted streams, are visited. Based on this methodology, the destination combines the source signal and the partial relay signal to perform the detection step. We show, in both simulation and hardware verification on the WARP platform, that using the CPD approach, the relay can avoid the considerable overhead of MIMO detection while helping the source-destination link to improve its performance.

Keywords - Communication Systems, MIMO Systems, Signal Processing
\end{abstract}

\section{INTRODUCTION}

Cooperative communications, where a relay node helps a direct source-destination communication, has been known to improve the performance by increasing the diversity in the destination [1], [2]. Multiple-input multiple-output (MIMO) systems have also been known to improve the reliability and data rate in point-to-point communications [3]. Because of the important role of MIMO systems in wireless standards, various detection algorithms and architectures, mostly based upon sphere detection, have been proposed to reduce the complexity of detection in MIMO systems [4], [5], [6], [7]. More recently, there have been some attempts to study the theoretical benefits and bounds on deploying MIMO nodes in cooperative scenarios, both as relays and as source/destination pairs. In doing so, lower bounds and upper bounds for MIMO relay networks were given in [8], [9].

While dedicated multi-antenna relays will be capable of performing computationally intensive operations, other potential MIMO relays will be mobile multi-antenna users that could choose to assist the active links in the environments during their idle times. Full detect-and-forward in the relay can require a significant amount of resources in MIMO cooperative communications, particularly if the relay chooses to perform a close-to-optimum detection. This effect becomes more important when one considers the practical resource constraints of idle MIMO users operating as relays. Therefore, it is crucial to distribute the detection task between the relay and the destination in such a way that the relay does not need to spend too much of its processing and transmit power, and yet, can enhance the performance compared to a non-relay scenario.

In order to address these challenges, we propose novel cooperative partial detection schemes in MIMO relay channels, where instead of applying the conventional full detection in the relay, the relay performs a partial detection and forwards the detected parts of the message to the destination. We will show that this cooperative detection scheme improves the performance compared to non-relay scenarios with limited computational overhead in the relay, and helps in distributing the detection process between the relay and destination. Finally, we provide an over-the-air implementation of this technique on the WARP platform [10].

This paper is organized as follows: Section II covers the system model definition. The full detect and forward scheme is described in section III. The proposed cooperative partial detection algorithm is presented in section IV. Monte-Carlo simulation results and hardware verification of the proposed scheme are presented in section $\mathrm{V}$.

\section{SySTEM MODEL}

We assume a three node network: the source, relay and destination, denoted by $\mathrm{S}, \mathrm{R}$ and $\mathrm{D}$; respectively. We further assume that the source, relay and destination are equipped with $M_{s}, M_{r}$ and $M_{d}$ antennas; respectively. Given the practical limitations of deploying full duplex radios, we assume the relay operates in half-duplex mode. The communication between the source and the destination is performed in two time slots. In the first time slot, the source broadcasts its message to both the relay and the destination; and in the second time slot, the relay, using a $M_{r u} \leq M_{r}$ subset of its antennas, transmits its message to the destination while the source is silent.

The received signals at the relay and destination at the end of the first time slot are given by

$$
\begin{aligned}
\mathbf{y}_{r} & =\mathbf{H}_{s r} \mathbf{x}_{s}+\mathbf{n}_{r}, \\
\mathbf{y}_{d}^{(1)} & =\mathbf{H}_{s d} \mathbf{x}_{s}+\mathbf{n}_{d}^{(1)} .
\end{aligned}
$$

Likewise, the received signal at the destination at the end of the second time slot is given by

$$
\mathbf{y}_{d}^{(2)}=\mathbf{H}_{r d} \mathbf{x}_{r}+\mathbf{n}_{d}^{(2)}
$$

where superscripts ${ }^{(1)}$ and ${ }^{(2)}$ are used to distinguish the first and second time slots. Since the relay receives only at the end of the first time slot, no superscript is used for the relay. In Eq. (1) to (3), $M_{r u}$ corresponds to the number of utilized antennas in the relay during the second time slot; hence $M_{r u} \leq M_{r}$. The noise vectors, $\mathbf{n}_{r}, \mathbf{n}_{d}^{(1)}$ and $\mathbf{n}_{d}^{(2)}$ are of size $M_{r}, M_{d}$ and $M_{d}$, with each of their elements chosen from a complex symmetric Gaussian variable $\mathcal{C} N(0,1)$. We also assume that each element of the $\mathbf{x}_{s}, \mathbf{x}_{r}$ and $\mathbf{x}_{d}$ vectors are chosen from a QAM modulation, $\Omega$, with the modulation set size of $w=|\Omega|$, and average power constraint of $E\left[x_{i}{ }^{2}\right]=1$. Also, note that since we do not assume any channel coding, the receivers perform only hard detection.

As illustrated in Figure 1, the $\mathbf{H}_{s r}, \mathbf{H}_{r d}$ and $\mathbf{H}_{s d}$ are matrices of sizes $M_{r} \times M_{s}, M_{d} \times M_{r u}$ and $M_{d} \times M_{s}$; and correspond to the channel matrices between the source and the relay, relay and the destination, and source and the destination, respectively. All these channel matrices have independent elements, each drawn from a circularly symmetric Gaussian random distribution with zero mean and variances of $\frac{\mathrm{SNR}_{s r}}{M_{s}}, \frac{\mathrm{SNR}_{r d}}{M_{r u}}$ and $\frac{\mathrm{SNR}_{s d}}{M_{s}}$, respectively. We make the 
practically feasible assumption that the $\mathbf{H}_{s r}$ matrix is known at the relay; and $\mathbf{H}_{s d}$ and $\mathbf{H}_{r d}$ matrices are known at the destination node; thus, only the receivers of each communication link have complete channel knowledge.

The signal-to-noise ratios, SNR, at each of the receive antennas of the relay and destination are defined as

$$
\mathrm{SNR}_{s r}=\frac{\mu P}{\left(d_{s r}\right)^{\alpha}}, \mathrm{SNR}_{s d}=\frac{\mu P}{\left(d_{s d}\right)^{\alpha}}, \mathrm{SNR}_{r d}=\frac{(1-\mu) P}{\left(d_{r d}\right)^{\alpha}}
$$

where $\alpha$ is the path loss exponent, and usually changes between 2 and 6 . The above SNR equations imply that the sum transmit power from the source and the relay is set to $P$, and is split with a proportion factor of $0<\mu \leq 1$, such that the source uses $\mu P$ and the relay uses $(1-\mu) P$.

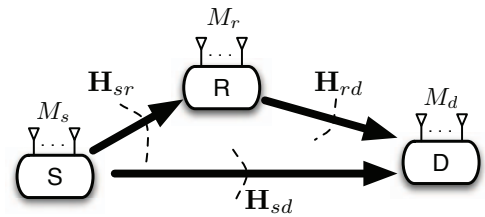

Fig. 1. A relay network with three nodes: source, relay and destination. The respective channel matrices are denoted by $\mathbf{H}_{s r}, \mathbf{H}_{r d}$ and $\mathbf{H}_{s d}$.

\section{FULL DETECT-AND-FORWARD (FDF) WITH MIMO RELAYS}

In this section, we present the symbol-level detector in the relay and destination. In the full detect-and-forward (FDF), the source transmits $\mathbf{x}_{s}$ in the first time slot, and the relay and destination receive their copy of the transmitted vector, $\mathbf{y}_{r}$ and $\mathbf{y}_{d}^{(1)}$. Then, the relay performs sphere detection on its received vector, $\mathbf{y}_{r}$, to find $\tilde{\mathbf{x}}_{s}$, where $\tilde{\mathbf{x}}_{s}$ is equal to $\mathbf{x}_{s}$ in an error-free detection:

$$
\tilde{\mathbf{x}}_{s}=\underset{\mathbf{b} \in \mathcal{O}^{m}}{\operatorname{argmin}}\left\|\mathbf{y}_{r}-\mathbf{H}_{s r} \mathbf{b}\right\|^{2}
$$

The norm in (5) can be re-written as [4]

$$
\begin{aligned}
D(\mathbf{b}) & =\left\|\mathbf{y}_{r}-\mathbf{H}_{s r} \mathbf{b}\right\|^{2} \\
& =\left\|\mathbf{Q}^{*} \mathbf{y}_{r}-\mathbf{R} \mathbf{b}\right\|^{2}=\sum_{i=M_{s}}^{1}\left|y_{i}{ }^{\prime}-\sum_{j=i}^{M_{s}} R_{i, j} b_{j}\right|^{2}
\end{aligned}
$$

where $\mathbf{H}_{s r}=\mathbf{Q R}, \mathbf{Q Q}^{*}=\mathbf{I}$ and $\mathbf{y}^{\prime}=\mathbf{Q}^{*} \mathbf{y}_{r}$. Throughout this paper, we will use the superscript ${ }^{*}$ to denote the matrix Hermitian transpose. This minimization process can be performed in a depthfirst tree search [4].

Finally, the relay transmits the $\mathbf{x}_{r}=\tilde{\mathbf{x}}_{s}$ in the second time slot to the destination, using the same modulation order. The received vector at the destination from the relay is denoted by $\mathbf{y}_{d}^{(2)}$.

Given the two received copies in the destination, the MaximumLikelihood detector is equivalent to

$$
\underset{\mathbf{x} \in \mathcal{O}^{M_{s}}}{\operatorname{argmin}}\left(\left\|\mathbf{y}_{d}^{(2)}-\mathbf{H}_{r d} \mathbf{x}\right\|_{1}^{2}+\left\|\mathbf{y}_{d}^{(1)}-\mathbf{H}_{s d} \mathbf{x}\right\|_{2}^{2}\right) .
$$

After expanding each of the norms in (7) and regrouping the different terms, (7) can be rewritten as

$$
\underset{\mathbf{x} \in \mathcal{O}^{M_{s}}}{\operatorname{argmin}}\left(\left\|\mathbf{y}_{F D F}-\mathbf{H}_{F D F} \mathbf{x}\right\|_{2}^{2}\right)
$$

where the equivalent channel matrix, $\mathbf{H}_{F D F}$, and the equivalent received vector, $\mathbf{y}_{F D F}$, are given by

$$
\begin{array}{r}
\mathbf{H}_{F D F}=\left(\mathbf{H}_{s d}^{*} \mathbf{H}_{s d}+\mathbf{H}_{r d}^{*} \mathbf{H}_{r d}\right)^{1 / 2} \\
\mathbf{y}_{F D F}=\mathbf{H}_{F D F}^{-1}\left(\mathbf{H}_{s d}^{*} \mathbf{y}_{d}^{(1)}+\mathbf{H}_{r d}^{*} \mathbf{y}_{d}^{(2)}\right) .
\end{array}
$$

The destination can now perform sphere detection on the newly formed combined matrix and vector of (9) and (10). It should be pointed out that a similar approach to performing the detection process is to concatenate the two received vectors rather than directly computing the (9) and (10). While that approach avoids the combining step, it requires a more complex pre-processing stage since the QR decomposition will be performed on matrices with larger sizes.

The full detect-and-forward strategy, discussed in this section requires a relay with a considerable amount of resources to perform full detection of the source signal. While this can be feasible for dedicated and infrastructure relays, this may not be a practical assumption for mobile resource-limited, e.g. battery-operated, devices that may choose to operate as relays when they are in idle modes. In such scenarios, depending on the resource availability in the relay, the relay may not be capable of dedicating sufficient resources to other communication links. Therefore, it is critical to develop lowoverhead strategies that a resource-limited relay can use to assist another communication link while preserving sufficient resources given its resource budget.

\section{Cooperative Partial Detection (CPD) with MiMo} RELAYS

In this section, we propose cooperative partial detection (CPD) as a low-complexity strategy for relays with limited resources. The cooperative partial detection (CPD) is based on partial sphere detection in the relay to facilitate the cooperative detection strategy.

\section{A. Partial Sphere Detection (P-SD) in the Relay}

In order to reduce the relay overhead, we propose partial sphere detection (P-SD), where the relay visits only a subset of the tree levels as opposed to all the levels. Our proposed partial sphere detection (P-SD) requires similar pre-processing operations as that of the conventional sphere detector: the $\mathrm{QR}$ decomposition triangularizes the channel matrix, and the tree traversal starts from the top level, $i=M_{s}$, where $M_{s}$ is the number of transmit antennas. Unlike the conventional sphere detection method, the tree traversal of the partial sphere detection method terminates in one of the middle levels, and the corresponding minimum distance at that level is considered as the partial detected symbol vector. We call the number of visited antennas the expansion factor, ef, and, as pointed out in section II, use ef antennas of the relay to transmit those messages. Figure 2 shows this process for an example case with 16-QAM modulation, and expansion factor of 2 .

In other words, instead of transmitting $\mathbf{x}_{r}=\tilde{\mathbf{x}}_{s}$, as in FDF, the relay now transmits only ef symbols, $\mathbf{x}_{r}=\left[\tilde{x}_{1}, \ldots, \tilde{x}_{e f}\right]^{T}$, where the superscript ${ }^{T}$ denotes the vector transpose operation.

In order to understand the computational savings of the P-SD, we should note that the complexity of sphere detection, in terms of computation count, can be modeled as: $C_{S D}=\sum_{i=M_{s}}^{1} C_{i} E\left\{D_{i}\right\}$, where $C_{i}$ corresponds to the computation count for one node in level $i$, and $E\left\{D_{i}\right\}$ is the average number of visited nodes in level $i$. Based on Eq. (6), it is clear that $C_{i}$ is larger for the nodes closer to the bottom of the tree, i.e. $C_{i+1}<C_{i}$. Therefore, $\mathrm{P}$-SD reduces the total complexity in the relay by not only reducing the total number of visited nodes, but also by limiting the search to the nodes located at the top of the tree with less computation per node. It should be pointed out that since the channel matrix changes at a slower rate than the received vector, the $\mathrm{QR}$ decomposition needs to happen at a slower rate. Using interpolation methods for OFDM systems can further reduce the $\mathrm{QR}$ overhead. Therefore, for many practical systems, the computations of $C_{S D}$ dominate the overall complexity of the detector. 


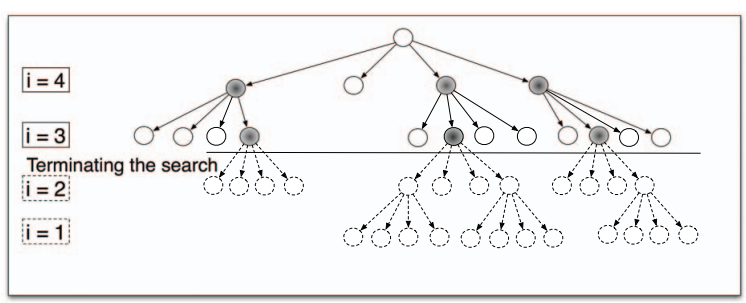

Fig. 2. The tree structure for a partial sphere detector with the expansion factor of two, ef $=2$. Each node has 4 children for the example case of 4-QAM modulation.

\section{B. Detection in the Destination: MRC Cooperative Partial Detection} (MRC-CPD)

We present the detection scheme in the destination. The destination combines the two received vectors, $\mathbf{y}_{d}^{(1)}$ and $\mathbf{y}_{d}^{(2)}$, as shown below.

We first break the original transmitted vector into two parts:

$$
\mathbf{x}_{s}=\mathbf{x}=\left[\mathbf{x}_{1}, \mathbf{x}_{2}\right]^{T}
$$

where

$$
\mathbf{x}_{1}=\left[x_{1}, \ldots, x_{e f}\right]^{T}, \mathbf{x}_{2}=\left[x_{e f+1}, \ldots, x_{M_{s}}\right]^{T},
$$

and denote the relay's transmitted vector as

$$
\mathbf{x}_{r}=\tilde{\mathbf{x}}_{1}=\left[\tilde{x}_{1}, \ldots, \tilde{x}_{e f}\right]^{T} .
$$

We also split the source-destination channel matrix into two parts according to (11):

$$
\mathbf{H}_{s d}=\left[\begin{array}{ll}
\mathbf{H}_{1} & \mathbf{H}_{2}
\end{array}\right] .
$$

Similar to Eq. (7), assuming perfect detection in the relay, i.e. $\tilde{\mathbf{x}}_{1}=$ $\mathbf{x}_{1}$, the symbol level maximum-likelihood solution can be written as the following minimization problem:

$$
\begin{aligned}
& \underset{\mathbf{x} \in \mathcal{O}^{M_{s}}}{\operatorname{argmin}}\left(\left\|\mathbf{y}_{d}^{(2)}-\mathbf{H}_{r d} \mathbf{x}_{1}\right\|_{2}^{2}+\left\|\mathbf{y}_{d}^{(1)}-\mathbf{H}_{1} \mathbf{x}_{1}-\mathbf{H}_{2} \mathbf{x}_{2}\right\|_{2}^{2}\right)= \\
& \underset{\mathbf{x} \in \mathcal{O}^{M_{s}}}{\operatorname{argmin}}\left(A-B-B^{*}+g\left(\mathbf{y}_{d}^{(1)}, \mathbf{y}_{d}^{(2)}\right)\right),
\end{aligned}
$$

where $g($.$) contains those terms that do not depend on \mathbf{x}$ and, hence, will not affect the solution, and $A$ and $B$ are given by:

$$
\begin{aligned}
A & =\mathbf{x}_{2}^{*} \mathbf{H}_{2}^{*} \mathbf{H}_{2} \mathbf{x}_{2}+\mathbf{x}_{1}^{*} \mathbf{H}_{r d}^{*} \mathbf{H}_{r d} \mathbf{x}_{1}+\mathbf{x}_{1}^{*} \mathbf{H}_{1}^{*} \mathbf{H}_{1} \mathbf{x}_{1} \\
+ & \mathbf{x}_{1}^{*} \mathbf{H}_{1}^{*} \mathbf{H}_{2} \mathbf{x}_{2}+\mathbf{x}_{2}^{*} \mathbf{H}_{2}^{*} \mathbf{H}_{1} \mathbf{x}_{1} \\
B & =\mathbf{x}_{2}^{*} \mathbf{H}_{2}^{*} \mathbf{y}_{d}^{(1)}+\mathbf{x}_{1}^{*} \mathbf{H}_{1}^{*} \mathbf{y}_{d}^{(1)}+\mathbf{x}_{1}^{*} \mathbf{H}_{r d}^{*} \mathbf{y}_{d}^{(2)} \\
& =\mathbf{x}_{1}^{*}\left(\mathbf{H}_{1}^{*} \mathbf{y}_{d}^{(1)}+\mathbf{H}_{r d}^{*} \mathbf{y}_{d}^{(2)}\right)+\mathbf{x}_{2}^{*} \mathbf{H}_{2}^{*} \mathbf{y}_{d}^{(1)}
\end{aligned}
$$

Comparing (15) with

$$
\begin{aligned}
& \left\|\mathbf{y}_{C P D}-\mathbf{H}_{C P D} \mathbf{x}\right\|_{2}^{2}= \\
& \left\|\mathbf{y}_{C P D}\right\|_{2}^{2}-\mathbf{x}^{*} \mathbf{H}_{C P D}^{*} \mathbf{y}_{C P D}-\mathbf{y}_{C P D}^{*} \mathbf{H}_{C P D} \mathbf{x} \\
& +\mathbf{x}^{*} \mathbf{H}_{C P D}^{*} \mathbf{H}_{C P D} \mathbf{x}
\end{aligned}
$$

shows that the original problem in (15) is equivalent to

$$
\underset{\mathbf{x} \in \mathcal{O}^{M_{s}}}{\operatorname{argmin}}\left(\left\|\mathbf{y}_{C P D}-\mathbf{H}_{C P D} \mathbf{x}\right\|_{2}^{2}\right)
$$

if we set

$$
\mathbf{H}_{C P D}=\left[\begin{array}{cc}
\mathbf{H}_{1}^{*} \mathbf{H}_{1}+\mathbf{H}_{r d}^{*} \mathbf{H}_{r d} & \mathbf{H}_{1}^{*} \mathbf{H}_{2} \\
\mathbf{H}_{2}^{*} \mathbf{H}_{1} & \mathbf{H}_{2}^{*} \mathbf{H}_{2}
\end{array}\right]^{1 / 2}
$$

$$
\mathbf{y}_{C P D}=\mathbf{H}_{C P D}^{-1}\left[\begin{array}{c}
\mathbf{H}_{1}^{*} \mathbf{y}_{d}^{(1)}+\mathbf{H}_{r d}^{*} \mathbf{y}_{d}^{(2)} \\
\mathbf{H}_{2}^{*} \mathbf{y}_{d}^{(1)}
\end{array}\right] .
$$

After combining the effective $\mathbf{y}_{C P D}$ and $\mathbf{H}_{C P D}$, they are passed to a sphere detector to detect the ML solution $\mathrm{x}$. Because of the similarity of this combining scheme and the receiver diversity maximal ratio combining (MRC) in SIMO systems, we call this symbol level combining MRC Cooperative Partial Detection (MRCCPD).

\section{Performance COMPARison}

In this section, we compare the BER performance of the proposed detectors using both Monte-Carlo simulations and the Wireless Openaccess Research Platform (WARP).

\section{A. Simulation Results}

We assume a three node relay network topology with the relay located between the source and destination, on the same line, and thus $d_{s d}=d_{s r}+d_{r d}$, and a path loss exponent of $\alpha=3$. We assume a fixed location for the relay, and then optimize the performance by varying the power splitting ratio $\mu$, as defined in Eq. (4), from the discrete set of $\{0.1,0.2, \ldots, 0.9\}$, and use the one with the highest performance, i.e. $\mu=\mu_{F D F}$. In order to make sure that the savings in the relay are not limited to baseband processing savings, we also limit the transmit power of the relay for the CPD cases:

$$
\mu_{C P D}^{(e f)}=1-\left(1-\mu_{F D F}\right) e f / M_{s},
$$

which guarantees that by picking the partial detection strategy, the relay not only saves in the baseband computational processing, but also, in the total relay transmit power.

Figure 3 shows the BER performance for 4 antenna, 16-QAM modulation, and the ef values $e f=2$ and 3. In the CPD method, the relay chooses the ef streams based on the sorted $\mathrm{QR}$ technique.

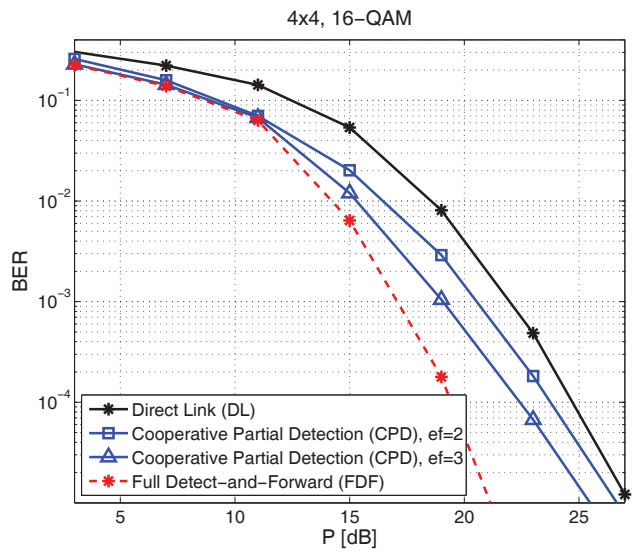

Fig. 3. BER Comparison for a system with $M_{s}=M_{d}=4$ and 16-QAM The relay is located at $d_{s r}=0.2$. The expansion factor is set to ef $=2$ and 3 , and the power splitting ratio of the FDF method is $\mu_{F D F}=0.6$.

\section{B. Hardware Verification}

In this section, we describe the hardware platform to perform cooperative communication tests. WARPLab allows rapid prototyping of physical layer algorithms over the air, by exposing WARP hardware [10] to MATLAB. In this setup, multiple WARP boards are connected to a host PC through an Ethernet switch. A set of boards are designated as the transmitters and a set of boards are designated as the receivers through the WARPLab framework. To transmit, raw samples (I/Q) values are generated in MATLAB and 
uploaded to the transmit boards via Ethernet. When the host PC sends a trigger signal to all nodes via Ethernet, the transmit boards modulate the raw samples in the FPGA transmit buffers to the 2.4 $\mathrm{GHz}$ frequency signals, and transmit them through the radio boards of the transmit boards. The receive boards capture the incoming RF signals, downconvert them to the baseband samples and store them in the receive buffers of the FPGA. The host PC, in MATLAB, reads and process the values in the receive buffers via Ethernet. Note that the relay's baseband physical layer is a highly parallel architecture, and therefore, can effectively utilize FPGA's resources. As part of the future work, we will transfer parts of these processing from the host PC to the FPGA.

The transmit signal models a narrowband single subcarrier system. Since the sampling frequency is $40 \mathrm{MHz}$, an I/Q vector generated in MATLAB is upsampled by 128 in MATLAB to generate a narrowband signal with bandwidth of $625 \mathrm{kHz}$. The generated transmit vector includes preamble for synchronization and space-time coded pilots for estimating the channel. Since all digital baseband processing is done in MATLAB on the host PC, the host processes the preamble to perform timing synchronization and pilots to estimate the channel.

Our test setup, Figure 4, is a $2 \times 2$ three node cooperative system. A total of three WARP boards are connected to a host computer through an Ethernet switch. Experiements are conducted using an Azimuth ACE 400 WB wireless channel emulator [11]. The emulator can support up to a $4 \times 4$ setup-it has four inputs and four outputs and 16 bidirectional links. For the $2 \times 2$ full MIMO relay setup, we use 2 inputs, 4 outputs and 12 paths. For the first time slot, we designate one node as the source, one node as the relay, and one node as the destination. Four forward links are used to connect the source node to the relay node. Similarly, four other links are used to connect the source node to the destination node. Since all processing is done at the host computer, we can use the reverse link (which can be independent from the forward link) for the relay to destination link. We designate one node as the relay and one node as the destination and connect the two nodes with four reverse links.

The hardware emulation results using the platform are shown in Figure 5 for a $2 \times 2,16$-QAM system, where the relay is located at $d_{s r}=0.5$, and the power splitting ratio is $\mu=0.5$, and the channel is a 3GPP Class B channel [11]. Since the tests are performed on a hardware platform, the performance curves take into account the effects of the baseband processing as well as the RF chain, e.g. the amplifiers, the AGC (automatic gain control), imperfect channel estimate, etc. In the presence of such effects, the CPD method provides a middle point that improves the performance compared to the no-relay scenario while avoiding the larger complexity of the FDF method, which conforms with the simulation results for other systems dimensions.

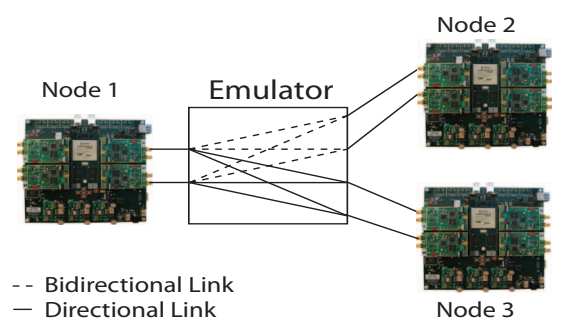

Fig. 4. Test setup using the WARP boards and the Azimuth channel emulator.

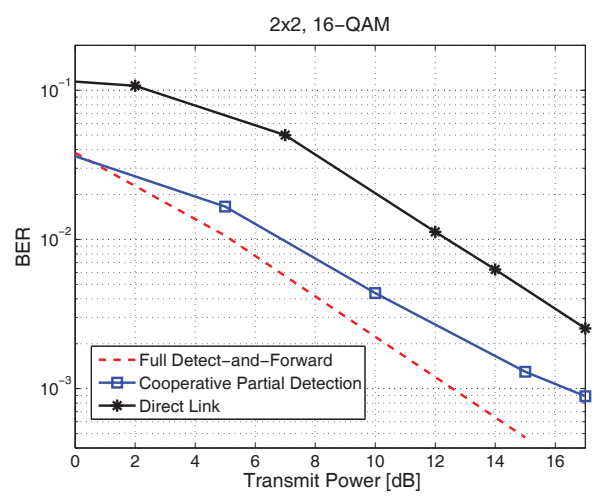

Fig. 5. BER comparison of the no-relay, CPD and FDF techniques using the WARP hardware platform at the $2.4 \mathrm{GHz}$ band. The channel emulation is done using the Azimuth ACE $400 \mathrm{WB}$ [11] channel emulator, and the results include the RF effects.

\section{CONCLUSION}

In this paper, we proposed a novel and practical cooperative partial detection scheme for MIMO relay networks, which was based on an architecture-friendly MIMO detection scenario. We showed that this scheme can be used to distribute the computational processing between the source and the destination. Furthermore, we verified this algorithm on hardware using the WARP platform.

\section{ACKNOWLEDGEMENT}

This work was supported in part by Xilinx, Azimuth Systems, and by NSF under grants CCF-0541363, CNS-0551692, EECS-0925942, CNS-0923479, and CNS-0619767. The authors also would like to thank the reviewers for their comments on improving the content of the paper.

\section{REFERENCES}

[1] A. Sendonaris, E. Erkip and B. Aazhang, "User cooperation diversity part I: System Discription," IEEE Trans. on Communications, vol. 51, no. 11, pp. 1927-1938, Nov. 2003.

[2] J. N. Laneman, D. N. C. Tse and G. W. Wornell, "Cooperative diversity in wireless networks: Efficient protocols and outage behavior," IEEE Trans. on Info Theory, vol. 50, no. 12, pp. 3062-3080, Dec. 2004.

[3] G. J. Foschini and M. J. Gans, "On limits of wireless communications in a fading environment when using multiple antennas," Wireless Personal Communications, vol. 6, pp. 311-335, 1998.

[4] E. Viterbo and J. Boutros, "A universal lattice decoder for fading channels," IEEE Trans. Inf. Theory, vol. 45, no. 5, Jul. 1999.

[5] M. Myllyla., M. Juntti and J. R. Cavallaro, "Architecture design and implementation of the increasing radius - List sphere detector algorithm," ICASSP, 2009.

[6] A. Burg, M. Borgmann, M. Wenk, M. Zellweger, W. Fichtner and H. Bolcskei, "VLSI implementation of MIMO detection using the sphere decoding algorithm," IEEE JSSC, vol. 40, no. 7, pp. 1566-1577, Jul. 2005.

[7] K. Amiri, P. Radosaljevic and J. R. Cavallaro, "Architecture and algorithm for a stochastic soft-output MIMO detector," 4lth Asilomar Conf on Signals, Systems and Computers, pp. 1034-1038, Oct./Nov. 2007.

[8] B. Wang, J. Zhang and A. Host-Madsen, "On the capacity of MIMO relay channels," IEEE Trans. on Info Theory, vol. 51, no. 1, pp. 29-43, Jan. 2005.

[9] C. K. Lo, S. Vishwanath and R. W. Heath, "Rate bounds for MIMO relay channels using precoding," IEEE Global Telecommunications Conference, Nov.-2 Dec. 2005.

[10] K. Amiri, Y. Sun, P. Murphy, C. Hunter, J. Cavallaro, and A. Sabharwal, "Warp, a unified wireless network testbed for education and research," in IEEE International Conference on Microelectronic Systems Education, 2007., June 2007, pp. 53-54.

[11] "Azimuth Systems : http://www.azimuthsystems.com/." 